\title{
Banana regime pressure anisotropy in a bumpy cylinder magnetic field
}

\author{
A. L. García-Perciante, J. D. Callen, K. C. Shaing, and C. C. Hegna \\ University of Wisconsin-Madison, Madison, Wisconsin 53706-1609
}

(Received 8 August 2005; accepted 29 November 2005; published online 20 January 2006)

\begin{abstract}
The pressure anisotropy is calculated for a plasma in a bumpy cylindrical magnetic field in the low collisionality (banana) regime for small magnetic-field modulations $(\epsilon \equiv \Delta B / 2 B \ll 1)$. Solutions are obtained by integrating the drift-kinetic equation along field lines in steady state. A closure for the local value of the parallel viscous force $\mathbf{B} \cdot \nabla \cdot \boldsymbol{\Pi}_{\|}$is then calculated and is shown to exceed the flux-surface-averaged parallel viscous force $\left\langle\mathbf{B} \cdot \nabla \cdot \boldsymbol{\Pi}_{\|}\right\rangle$by a factor of $\mathcal{O}(1 / \epsilon)$. A high-frequency limit $(\omega \gg \nu)$ for the pressure anisotropy is also determined and the calculation is then extended to include the full frequency dependence by using an expansion in Cordey eigenfunctions. (C) 2006 American Institute of Physics. [DOI: 10.1063/1.2159168]
\end{abstract}

\section{INTRODUCTION}

In toroidal magnetic-field configurations, $|B|$ variations within flux surfaces produce viscous damping forces on the plasma. Most calculations accounting for this parallel viscous damping introduce a flux-surface-average procedure. However, if one is interested in using closure relations for simulations of fluid equations, a local expression for the viscous force is required. While rigorous expressions for the evolution of the flux-surface-averaged quantities exist, applicability of this effect is limited to the evolution of equilibrium quantities. Additionally, the variation of viscous forces within flux surfaces may introduce new physics that the fluxsurface-averaging procedure eliminates. A large varying component of $\mathbf{B} \cdot \nabla \cdot \boldsymbol{\Pi}_{\|}$yields an anisotropic damping of parallel flows. The quantification of this effect is required, for example, to address the suppression of fluctuations through shear flow generation as a dynamic and space-dependent process.

Closures for the flux-surface-averaged parallel viscous force are available. The calculation was originally carried out for steady-state conditions. ${ }^{1-5}$ It was recognized by Hirshman $^{6}$ that this closure yields inconsistencies if one is interested in a temporally evolving parallel force balance. Because of this, a dynamic closure was calculated by various authors. ${ }^{7-11}$ Initial condition effects were introduced in Ref. 11 and the explicit dependence of the closure on time, including initial conditions, has been addressed recently. ${ }^{10}$ With this type of dynamic closure, some selected phenomena can be addressed formally, for example, the damping of the parallel flow variable $U \equiv V_{\|} / B$ which is a flux-surface quantity.

However, for nonlocal phenomena, the parallel viscous force is often approximated by a flux-surface-averaged closure $\left\langle\mathbf{B} \cdot \nabla \cdot \boldsymbol{\Pi}_{\|}\right\rangle$, i.e., neglecting its variation within a flux surface. In this regard, we show that approximating the viscosity by its averaged value is far from realistic. By neglecting the spatial variations of $\mathbf{B} \cdot \nabla \cdot \boldsymbol{\Pi}_{\|}$and working with a flux-surface-averaged closure, one misses the largest part of the closure. Specifically, we find $\mathbf{B} \cdot \nabla \cdot \Pi_{\|} /\left\langle\mathbf{B} \cdot \nabla \cdot \Pi_{\|}\right\rangle$ $\sim \mathcal{O}(1 / \epsilon)$, where $\epsilon \equiv \Delta B / 2 B \ll 1$ represents the small modu- lation of the magnetic-field strength along a field line. This result justifies the need for a local closure.

Wang and Callen ${ }^{5}$ calculated part of the poloidal variation of the parallel viscous force due to the term that gives rise to the averaged $\left\langle\mathbf{B} \cdot \nabla \cdot \boldsymbol{\Pi}_{\|}\right\rangle$. Also, a generalized stress tensor was formulated recently for arbitrary collisionality. ${ }^{12}$ Both calculations were carried out under a steady-state assumption. Hsu et al. ${ }^{9}$ provided some insight into the frequency-dependent complete spatial variation by suggesting a procedure for its calculation in an axisymmetric configuration. Here, the local nature of the viscous force is derived in the low-collision-frequency (banana) regime for a model bumpy cylinder magnetic field. We calculate the local closure for the steady-state case by directly integrating the relevant drift-kinetic equation along field lines. With the lowest-order correction to a Maxwellian distribution function that has a nonvanishing $p_{\|}-p_{\perp}$ moment, we calculate the pressure anisotropy and the parallel viscous force. The generalization to a dynamic closure is explored towards the end of this paper. An expression for the pressure anisotropy is obtained formally and the result is expressed in terms of Cordey eigenfunctions. ${ }^{13}$

In Sec. II, a Fourier expansion is used to approximate the ordering of the local $\mathbf{B} \cdot \nabla \cdot \Pi_{\|}$compared with the usual averaged closure. The local closure for $p_{\|}-p_{\perp}$ is calculated in Sec. III by solving the drift-kinetic equation to second order in the collisionality parameter $\nu_{*} \equiv \nu_{e} \epsilon^{3 / 2} / \omega_{b} \ll 1$. The parallel viscous force is calculated in Sec. IV. The calculation of the high-frequency limit for the pressure anisotropy is carried out in Sec. V, and Sec. VI is devoted to extending the calculation to a full dynamic situation for all values of $\omega / \nu$. A summary and final remarks are included in Sec. VII. Specific details for some key steps in the derivation of the closures are shown in Appendices A and B.

\section{MOTIVATION: MAGNITUDE OF B. $\nabla \cdot \Pi_{\|}$}

In neoclassical theory, fluid moment equations include viscous forces. In particular, a closure for the stress tensor which is defined as 


$$
\boldsymbol{\Pi}=\int d^{3} v m\left(\mathbf{v v}-\frac{1}{3} v^{2} \mathbf{I}\right) f,
$$

is required to close the system of fluid moment equations describing the plasma dynamics. In a small gyroradius ordering $(\rho / \ell \ll 1)$ the stress tensor can be approximated by its parallel component which, to lowest order, has the ChewGoldberger-Low form:

$$
\boldsymbol{\Pi}_{\|}=\left(p_{\|}-p_{\perp}\right)(\mathbf{b b}-\mathbf{I} / 3) \equiv \Pi_{\|}(\mathbf{b} \mathbf{b}-\mathbf{I} / 3) .
$$

The last equality defines the "scalar" stress, i.e., the pressure anisotropy due to the inhomogeneous magnetic field in the direction $\mathbf{b} \equiv \mathbf{B} / B$ :

$$
\Pi_{\|}=p_{\|}-p_{\perp}=m \int d^{3} v\left(v_{\|}^{2}-\frac{v_{\perp}^{2}}{2}\right) f .
$$

To calculate the magnitude of the viscous force, we calculate the divergence of the stress tensor $\Pi \sim \boldsymbol{\Pi}_{\|}$as follows:

$$
\nabla \cdot \Pi_{\|}=\left(\nabla \Pi_{\|}\right) \cdot(\mathbf{b b}-\mathbf{I} / 3)+\Pi_{\|} \nabla \cdot \mathbf{b b} .
$$

Using tensor identities and assuming that the magnetic-field strength is modulated along a field line, the viscous force is calculated from

$$
\begin{aligned}
\nabla \cdot \Pi_{\|}= & \mathbf{b}\left(\mathbf{b} \cdot \nabla \Pi_{\|}\right)-\nabla \Pi_{\|} / 3 \\
& -\Pi_{\|}\left[\mathbf{b}\left(\frac{\mathbf{B} \cdot \nabla B}{B^{2}}\right)+\mathbf{b} \times(\nabla \times \mathbf{b})\right] .
\end{aligned}
$$

The parallel viscosity is obtained by projecting Eq. (5) in the B direction. This yields the simple expression

$$
\mathbf{B} \cdot \nabla \cdot \Pi_{\|}=\frac{2}{3}(\mathbf{B} \cdot \nabla) \Pi_{\|}-\Pi_{\|} \mathbf{b} \cdot \nabla B=\frac{2}{3} B \frac{\partial \Pi_{\|}}{\partial \ell}-\Pi_{\|} \frac{\partial B}{\partial \ell},
$$

where for the second equality we used $\mathbf{b} \cdot \nabla=\partial / \partial \ell$ assuming that the magnetic field varies along $\ell$ (distance along a field line); in the following, we assume a bumpy cylinder magnetic-field model given by

$$
B(\ell)=B_{\min }+\Delta B \sin ^{2}\left(\frac{\pi \ell}{L}\right),
$$

where $\Delta B=B_{\max }-B_{\min } \equiv 2 B_{0} \epsilon$, and for $\epsilon \ll 1$ we can write

$$
B \sim B_{0}\left[1+2 \epsilon \sin ^{2}\left(\frac{\pi \ell}{L}\right)\right] .
$$

This simple model has the advantage of simulating the gradients of the magnetic-field strength in the parallel (poloidal) direction in an axisymmetric field geometry while isolating the $\nabla B$ trapping effects. The modulation $\epsilon$ corresponds to the inverse aspect ratio $\left(\epsilon \simeq r / R_{0} \ll 1\right)$ in a toroidal configuration and will be considered as a small parameter.

Taking the flux-surface average on both sides of Eq. (6), we obtain

$$
\left\langle\mathbf{B} \cdot \nabla \cdot \Pi_{\|}\right\rangle=2 \epsilon \frac{\pi}{L} B_{0}\left\langle\Pi_{\|} \sin (2 \theta)\right\rangle,
$$

where

$$
\langle A\rangle \equiv \oint \frac{d \theta A}{B} / \oint \frac{d \theta}{B}
$$

and $\theta \equiv \pi \ell / L$. Because of the symmetry of the problem and the requirements for a physically relevant solution, the pressure anisotropy is continuous and finite within the field variation length. Thus, the flux-surface average of the first term in Eq. (6) vanishes. Combining Eq. (9) with the result from previous averaged calculations that derive the scaling $\left\langle\mathbf{B} \cdot \nabla \cdot \Pi_{\|}\right\rangle \sim \mathcal{O}(\sqrt{\epsilon})$, we can state

$$
\left\langle\Pi_{\|} \sin (2 \theta)\right\rangle \equiv \frac{L}{2 \pi} \int_{0}^{\pi} \Pi_{\|} \sin (2 \theta) d \theta \sim \mathcal{O}(1 / \sqrt{\epsilon}) .
$$

This does not imply any ordering for $\Pi_{\|}$itself. We still need to calculate the integral in Eq. (10) for which we assume a Fourier expansion of the form

$$
\Pi_{\|}=\sum_{n=1}^{\infty} a_{n} \sin (2 n \theta) .
$$

Here, the index $n$ is an odd integer since in order to have $\left\langle\Pi_{\|} \sin (2 \theta)\right\rangle \neq 0$ the pressure anisotropy has to be an odd function of $\vartheta$. The coefficients in the series are calculated from

$$
a_{n}=\frac{1}{2 \pi} \int_{0}^{\pi} \Pi_{\|} \sin (2 n \theta) d \theta .
$$

Equation (9) can be written as

$$
\left\langle\mathbf{B} \cdot \nabla \cdot \Pi_{\|}\right\rangle \simeq 2 \epsilon \frac{B_{0}}{2 L} \int_{0}^{\pi} \Pi_{\|} \sin (2 \theta) d \theta,
$$

which gives the first coefficient of the series,

$$
a_{1}=\frac{1}{2 \epsilon} \frac{L}{\pi} \frac{1}{B_{0}}\left\langle\mathbf{B} \cdot \nabla \cdot \Pi_{\|}\right\rangle \simeq \frac{1}{\sqrt{\epsilon}} \frac{L}{2 \pi} \frac{2.24}{B_{0}} \nu m n U\left\langle B^{2}\right\rangle,
$$

where we have introduced the standard closure for small $\epsilon$ : $\left\langle\mathbf{B} \cdot \nabla \cdot \boldsymbol{\Pi}_{\|}\right\rangle \simeq 2.24 \nu \sqrt{\epsilon} U m n\left\langle B^{2}\right\rangle$, in which $U \equiv V_{\|} / B$. Thus, the pressure anisotropy is

$$
\Pi_{\|} \simeq \frac{1}{\sqrt{\epsilon}} \frac{L}{2 \pi} \frac{2.24}{B_{0}} \nu m n U\left\langle B^{2}\right\rangle \sin (2 \theta)+\sum_{n=3}^{\infty} a_{n} \sin (2 n \theta) .
$$

With this series representation of $\Pi_{\|}$we can calculate both terms in the last equality of Eq. (6). Introducing the parallel derivative of $\Pi_{\|}$, given by

$$
\begin{aligned}
\frac{\partial \Pi_{\|}}{\partial \ell}= & \frac{2}{\sqrt{\epsilon}} \frac{1.12}{B_{0}} \nu m n U\left\langle B^{2}\right\rangle \cos \left(\frac{2 \pi \ell}{L}\right) \\
& +2 \frac{\pi}{L} \sum_{n=3}^{\infty} a_{n} n \cos \left(\frac{2 n \pi \ell}{L}\right),
\end{aligned}
$$

and rearranging terms we obtain 


$$
\begin{aligned}
\mathbf{B} \cdot \nabla \cdot \Pi_{\|}= & 2.24 \nu m n U\left\langle B^{2}\right\rangle\left[\frac{2}{3 \sqrt{\epsilon}} \cos (2 \theta)-\sqrt{\epsilon} \sin ^{2}(2 \theta)\right] \\
& +2 B_{0} \frac{\pi}{L} \sum_{n=3}^{\infty} a_{n}\left[\frac{2}{3} n \cos (2 n \theta)\right. \\
& -2 \epsilon \sin (2 \theta) \sin (2 n \theta)] .
\end{aligned}
$$

For the second term one can approximate

$$
\frac{2}{3} n \cos (2 n \theta)-2 \epsilon \sin (2 \theta) \sin (2 n \theta) \simeq \frac{2}{3} n \cos (2 n \theta),
$$

since for large aspect ratio we can clearly consider $n \geqslant 3$ $\gg 2 \epsilon$. Comparing the terms in the first and second lines in Eq. (17), we conclude

$$
\begin{aligned}
\mathbf{B} \cdot \nabla \cdot \Pi_{\|} \simeq & \frac{1.49}{\sqrt{\epsilon}} \nu m n U\left\langle B^{2}\right\rangle \cos (2 \theta) \\
& +\frac{2}{3} B_{0} \frac{\pi}{L} \sum_{n=3}^{\infty} a_{n} n \cos (2 n \theta)+\mathcal{O}(\sqrt{\epsilon}) .
\end{aligned}
$$

Thus, to lowest order (in $\epsilon \ll 1$ ), we have

$$
\mathbf{B} \cdot \nabla \cdot \Pi_{\|} \simeq \frac{2 B}{3} \frac{\partial \Pi_{\|}}{\partial \ell}+\mathcal{O}(\epsilon)=\frac{2 B}{3} \frac{l}{\partial \ell}\left(p_{\|}-p_{\perp}\right)+\mathcal{O}(\epsilon) .
$$

The dominant contribution to the local $\mathbf{B} \cdot \nabla \cdot \boldsymbol{\Pi}_{\|}$is due to the spatial variations in the pressure anisotropy $\left[\partial \Pi_{\|} / \partial \ell\right.$ in Eq. (6)] and not to the parallel gradient of the magnetic-field magnitude $[\partial B / \partial \ell$ in Eq. (6)]. The term in Eq. (20) is precisely the one that has zero flux-surface average; thus, when one examines the dynamics of plasma flows with the fluxsurface-averaged viscous force as a drive, only the effects of the smallest component of the stress $\left[\left\langle\mathbf{B} \cdot \nabla \cdot \Pi_{\|}\right\rangle\right.$ $\left.=\left\langle\Pi_{\|}(\partial B / \partial \ell)\right\rangle\right]$ are accounted for.

We must mention that in this section it was shown only that the variations of the magnetic-field magnitude are negligible compared to the variations of the pressure anisotropy when calculating the parallel viscous force (in this model and for small $\epsilon$ ). That is, at this point we can only conclude that $\mathbf{B} \cdot \nabla \cdot \boldsymbol{\Pi}_{\|}$has at least one term of order $1 / \sqrt{\epsilon}$, but we cannot formally infer its particular order (in $\epsilon$ ). Nonetheless, it is clear that the variation of $\mathbf{B} \cdot \nabla \cdot \Pi_{\|}$within a flux surface locally is much larger than its flux-surface average.

\section{LOCAL PRESSURE ANISOTROPY}

In order to include the trapped-particle effects that give rise to the pressure anisotropy in the distribution function, we consider a small kinetic distortion $F$ to a flow-shifted Maxwellian distribution $f_{M}$, as was done in Refs. 5, 10, and 14. Expanding this correction in the standard banana regime collisionality parameter $\nu_{*}=\nu / \epsilon^{3 / 2} \omega_{b} \ll 1$, where $\nu$ and $\omega_{b}$ are the collision and bounce frequencies respectively, we have

$$
\Pi_{\|}=m \int d^{3} v\left(v_{\|}^{2}-\frac{v_{\perp}^{2}}{2}\right)\left(f_{M}+F_{0}+F_{1}+\cdots\right) .
$$

Since the Maxwellian lowest-order solution is isotropic in velocity space, the only part of the distribution function that contributes to the stress tensor is the kinetic correction $F$ $=F_{0}+F_{1}+\cdots$. Then, changing to speed and pitch angle variables $\left(v\right.$ and $\left.\lambda \equiv 2 \mu / v^{2}\right)$, we have

$$
\begin{aligned}
\Pi_{\|}= & -m \sum_{\varsigma} \varsigma \pi \int d v v^{5} \int \frac{B d \lambda}{v_{\|}}\left(\frac{3}{2} \lambda B-1\right) \\
& \times\left(F_{0}+F_{1}+\cdots\right),
\end{aligned}
$$

where $\varsigma \equiv v_{\|} /\left|v_{\|}\right|$has values of 1 and -1 . In the simple geometry we are considering and ignoring heat flux effects, $F$ is governed by $\mathrm{y}^{5,10}$

$$
\begin{aligned}
\frac{\partial F}{\partial t}+v_{\|} \mathbf{b} \cdot \nabla\left(F+\frac{m}{T} v_{\|} B U f_{M}\right)= & -C_{R}(F) \\
& +\frac{v_{\|}}{p} f_{M} \mathbf{b} \cdot \nabla \cdot \Pi_{\|},
\end{aligned}
$$

which is a simplified form of the plasma drift-kinetic equation recast under the Chapman-Enskog ansatz. ${ }^{14,15}$ The time derivative term will be ignored here but will be taken into account in Secs. VI and V. A discussion on the nature of the collisional term for this problem can be found in Refs. 10 and 16 where Eq. (23) is solved order by order. The first term of the correction, $F_{0}$, is calculated elsewhere ${ }^{4,5}$ for this $\nu t$ $\gg 1$ case and is given by

$$
F_{0}=U \frac{m}{T} f_{M}\left[-v_{\|} B+\frac{v \varsigma}{2} \frac{1}{f_{c}} \int_{\lambda}^{\lambda_{c}} \frac{\left\langle B^{2}\right\rangle d \lambda^{\prime}}{\langle\sqrt{1-\lambda B}\rangle} H\left(\lambda_{c}-\lambda\right)\right] .
$$

Here $H\left(\lambda_{c}-\lambda\right)$ is the Heaviside step function and $f_{c}$ is the flow-weighted fraction of circulating (untrapped) particles defined as

$$
f_{c}=\frac{3}{4}\left\langle B^{2}\right\rangle \int_{0}^{\lambda_{c}} \frac{\lambda d \lambda}{\langle\sqrt{1-\lambda B}\rangle} .
$$

For the small $\epsilon$ approximation we are considering $f_{c} \sim 1$ $+\mathcal{O}(\sqrt{\epsilon}) \simeq 1-1.46 \sqrt{\epsilon}{ }^{4}$

The lowest-order solution $F_{0}$ is odd in $v_{\|}$and thus does not contribute to the moment in Eq. (22). The first nonzero term in the $P_{2} \equiv\left(v_{\|}^{2}-v_{\perp}^{2} / 2\right) / v^{2}$ moment of the distribution function is $F_{1}$ which we here calculate by integrating the next order drift-kinetic equation along a field line, which yields

$$
F_{1} \simeq \int_{0}^{\ell} \frac{C_{R}\left(F_{0}\right)}{v_{\|}} d \ell^{\prime}+\frac{2}{3 B} \frac{f_{M}}{p} \Pi_{\|} .
$$

Here, we have neglected an integration constant which is shown to be small compared to other terms in Appendix A.

When taking the $P_{2}$ moment of Eq. (26) the second term clearly vanishes. (For an explicit calculation and a discussion 
of the functional form of $F_{1}$ see Ref. 16.) Then, using Eq. (24) for $F_{0}$ to lowest order in $\epsilon$, we calculate the first term as follows:

$$
\begin{aligned}
\int_{0}^{\ell} \frac{d \ell^{\prime}}{v_{\|}} C_{R}\left(F_{0}\right)= & -U f_{M} \frac{m}{T} \frac{\nu_{\perp}}{2}\left\{\zeta\left(B^{2}\right)-\left\langle B^{2}\right\rangle \frac{\zeta\left(v_{\|}\right)}{\left\langle v_{\|}\right\rangle}\right. \\
& \left.+\left\langle B^{2}\right\rangle \frac{\lambda}{\left\langle v_{\|}\right\rangle} \frac{v^{2}}{2}\left[\zeta\left(\frac{B}{v_{\|}}\right)-\frac{\zeta\left(v_{\|}\right)}{\left\langle v_{\|}\right\rangle}\left\langle\frac{B}{v_{\|}}\right\rangle\right]\right\},
\end{aligned}
$$

where we have defined the operator

$$
\zeta[A(\ell)]=\int_{0}^{\ell} \frac{d \ell^{\prime}}{B\left(\ell^{\prime}\right)} A\left(\ell^{\prime}\right)
$$

In order to progress further, an explicit form of $B(\theta)$ has to be specified. For any magnetic field whose magnitude varies along its direction we can write $(\theta \equiv 2 \pi \ell / L)$

$$
B(\theta)=B_{0}[1+2 \epsilon \tau(\theta)]
$$

where we consider $\tau(\theta)$ to be a continuous, periodic function that varies between 0 and 1 . Using this general form for the magnetic field and defining a pitch-angle variable $s$ given by

$$
\lambda=\frac{1}{B_{0}} \frac{s^{2}}{s^{2}+2 \epsilon}
$$

the parallel velocity can be calculated (from $v_{\|}=\varsigma v \sqrt{1-\lambda B}$ ) as

$$
v_{\|}=\varsigma v \frac{\sqrt{2 \epsilon}}{\left(2 \epsilon+s^{2}\right)^{1 / 2}} \sqrt{1+s^{2} \tau(\theta)} .
$$

Then, introducing the model given by Eq. (29) and changing the pitch-angle variable to $s$ in Eq. (27), we obtain

$$
\begin{aligned}
\int_{0}^{\theta} \frac{d \theta}{v_{\|}} C_{R}\left(F_{0}\right) \simeq & \left\langle B^{2}\right\rangle U f_{M} \frac{\nu_{\perp}}{v_{\mathrm{th}}^{2}}\left(\frac{\zeta\left[\sqrt{1+s^{2} \tau(\theta)}\right]}{\left\langle\sqrt{1+s^{2} \tau(\theta)}\right\rangle}\right. \\
& -\zeta\left\{[1+2 \epsilon \tau(\theta)]^{2}\right\} \\
& -\frac{1}{2 \epsilon} \frac{s^{2} / 2}{\left\langle\sqrt{1+s^{2} \tau(\theta)}\right\rangle}\left\{\zeta\left[\frac{1}{\sqrt{1+s^{2} \tau(\theta)}}\right]\right. \\
& \left.\left.-\frac{\zeta\left[\sqrt{1+s^{2} \tau(\theta)}\right]}{\left\langle\sqrt{1+s^{2} \tau(\theta)}\right\rangle}\left\langle\frac{1}{\sqrt{1+s^{2} \tau(\theta)}}\right\rangle\right\}\right) .
\end{aligned}
$$

The free-streaming term and the direct $\lambda$-derivative term [first two terms in Eq. (32)] are smaller in the $\epsilon$ expansion and can thus be neglected. An explicit verification of this is shown for the bumpy cylinder case in Appendix B. Thus, to lowest order in the $\epsilon \ll 1$ expansion, we obtain [see Appendix $\mathrm{B}$ for details on determining the results given in Eqs. (33) and (34) below]

$$
\begin{aligned}
\Pi_{\|} \simeq & \frac{1}{\sqrt{2 \epsilon}} m n U\left\langle B^{2}\right\rangle \int d^{3} v \frac{\nu_{\perp}}{v_{\mathrm{th}}^{2}} \frac{v^{2}}{3} \frac{f_{M}}{n} \\
& \times \int_{0}^{1} \frac{\left(s^{2}+2 \epsilon\right)^{-3 / 2}}{\sqrt{1+s^{2} \tau(\theta)}} \frac{s^{3}}{\left\langle\sqrt{1+s^{2} \tau(\theta)}\right\rangle} \\
& \times\left\{\frac{\zeta\left[\sqrt{1+s^{2} \tau(\theta)}\right]}{\left\langle\sqrt{1+s^{2} \tau(\theta)}\right\rangle}\left\langle\frac{B}{\sqrt{1+s^{2} \tau(\theta)}}\right\rangle\right. \\
& \left.-\zeta\left[\frac{B}{\sqrt{1+s^{2} \tau(\theta)}}\right]\right\} d s .
\end{aligned}
$$

For the bumpy cylinder magnetic-field model given by Eq. (7), the pressure anisotropy is given by

$$
\Pi_{\|} \simeq \frac{1}{\sqrt{\epsilon}} \frac{L}{B_{0}}\left\langle B^{2}\right\rangle \operatorname{Umnf}_{0} \int \frac{v^{2}}{3} \frac{\nu_{\perp}}{v_{\mathrm{th}}^{2}} \frac{f_{M}}{n} d^{3} v,
$$

where the $\ell$ dependence is buried in the function $f_{0}$ which is defined in terms of complete $(E, K)$ and incomplete $\left[F, E\left(\theta, s^{2}\right)\right]$ elliptic functions as follows:

$$
\begin{aligned}
f_{0} \equiv & \frac{3}{8 \sqrt{2}} \int_{0}^{1} \frac{d s}{\sqrt{1-s^{2} \sin ^{2} \theta}} \frac{1}{E\left(s^{2}\right)} \\
& \times\left[F\left(\theta, s^{2}\right)-\frac{E\left(\theta, s^{2}\right)}{E\left(s^{2}\right)} K\left(s^{2}\right)\right] .
\end{aligned}
$$

The term in brackets in Eq. (35) has singularities for $s$ $=1$ and $\theta=L / 2$, since both $F\left(\pi \ell / L, s^{2}\right)$ and $K\left(s^{2}\right)$ diverge at that point and the square root in the denominator that multiplies the bracket vanishes. This "point" in phase space corresponds to particles at the pitch-angle boundary reaching the field maximum (which is their turning point). However, the expression for the pressure anisotropy in Eq. (34) is continuous and finite as will be shown now.

In order to examine the behavior of the integrand in Eq. (35) in the vicinity of $s=1$, an integration by parts can be performed and yields

$$
\begin{aligned}
f_{0} \equiv & \frac{3}{8 \sqrt{2}}\left\{\int_{0}^{1} \frac{d s}{E\left(s^{2}\right)} \frac{E\left(\pi \ell / L, s^{2}\right)}{\left[1-s^{2} \sin ^{2}(\pi \ell / L)\right]^{3 / 2}}\right. \\
& \left.-\frac{E(\pi \ell / L, 1)}{\sqrt{1-\sin ^{2}(\pi \ell / L)}}\right\} .
\end{aligned}
$$

Indeed, both terms on the right side of Eq. (36) are singular at $\ell=L / 2$. However, both singularities are of the same order and cancel since for $\ell=L / 2$ we have

$$
\begin{gathered}
\left.\int_{0}^{1} \frac{d s}{E\left(s^{2}\right)} \frac{E\left(\pi \ell / L, s^{2}\right)}{\left[1-s^{2} \sin ^{2}(\pi \ell / L)\right]^{3 / 2}}\right|_{\theta \rightarrow \pi / 2} \\
\quad=\left.\int_{0}^{1} \frac{d s}{\left(1-s^{2}\right)^{3 / 2}} \sim \frac{s}{\sqrt{1-s^{2}}}\right|_{s \rightarrow 1}
\end{gathered}
$$

and 


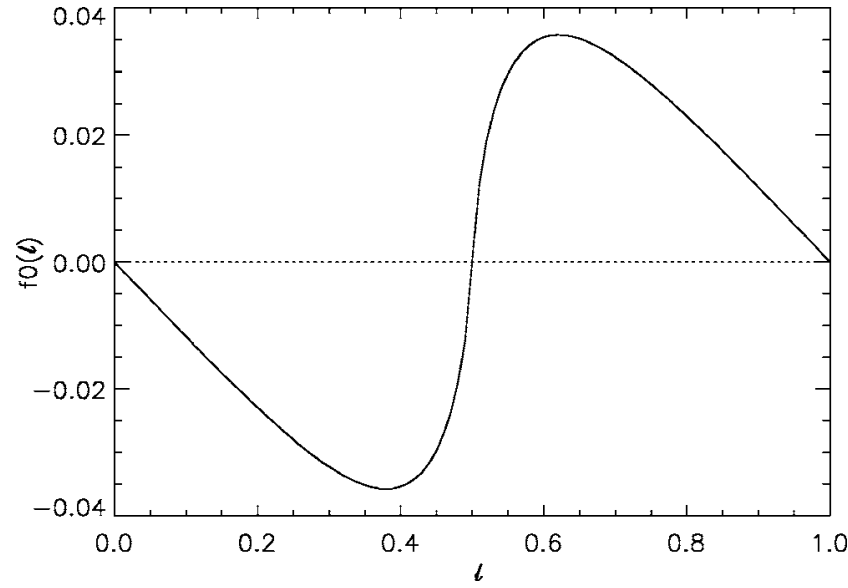

FIG. 1. The continuous spatial variation of $\Pi_{\|}$is given by the function $f_{0}$, where $\Pi_{\|} \propto \mathcal{O}\left(\epsilon^{-1 / 2}\right) U f_{0}(\theta)$.

$$
\frac{E(\pi \ell / L, 1)}{\sqrt{1-\sin ^{2}(\pi \ell / L)}}=\left.\tan \left(\frac{\pi \ell}{L}\right)\right|_{\theta \rightarrow \pi / 2} .
$$

That both singularities are the same can be easily seen by changing the variable $s \rightarrow \sin \theta$ in the far right side of Eq. (37). The functional form of $f_{0}(\theta)$ is shown in Fig. 1.

Both Eqs. (35) and (36) give well-behaved expressions for the variation of the pressure anisotropy as a function of $\ell$ consistent with the assumption in Sec. II. The continuity of $\Pi_{\|}$is thus guaranteed and no singularity problems should interfere with any numerical evaluation of the expressions obtained here.

\section{PARALLEL VISCOUS FORCE}

To calculate the parallel viscous force, we introduce the expression for $\Pi_{\|}$given in Eq. (34) in Eq. (6) which yields [for the bumpy cylinder model in Eq. (7)]

$$
\begin{aligned}
\mathbf{B} \cdot \nabla \cdot \Pi_{\|}= & \frac{L}{\sqrt{\epsilon}} m n U\left\langle B^{2}\right\rangle \int \frac{v^{2}}{3} \frac{\nu_{\perp}}{v_{\mathrm{th}}^{2}} \frac{f_{M}}{n} d^{3} v \\
& \times\left[\frac{2}{3} \frac{\partial f_{0}}{\partial \ell}+2 \epsilon \frac{\pi}{L} \sin \left(\frac{2 \pi \ell}{L}\right) f_{0}\right] .
\end{aligned}
$$

Using the flux-surface-averaged result

$$
\left\langle\mathbf{B} \cdot \nabla \cdot \boldsymbol{\Pi}_{\|}\right\rangle \equiv m n U\left\langle B^{2}\right\rangle \frac{f_{t}}{f_{c}} \int \frac{v^{2}}{3} \frac{\nu_{\perp}}{v_{\mathrm{th}}^{2}} \frac{f_{M}}{n} d^{3} v,
$$

we can write the unaveraged parallel viscous force in the following simple way:

$$
\mathbf{B} \cdot \nabla \cdot \Pi_{\|}=\left\langle\mathbf{B} \cdot \nabla \cdot \boldsymbol{\Pi}_{\|}\right\rangle\left(\frac{1}{\epsilon} f_{1}+f_{2}\right),
$$

where $f_{1}$ and $f_{2}$ are given by

$$
f_{1}=\frac{L}{1.46} \frac{2}{3} \frac{\partial f_{0}}{\partial \ell}, \quad f_{2}=\frac{2 \pi}{1.46} \sin \left(\frac{2 \pi \ell}{L}\right) f_{0},
$$

and we used the approximate value $f_{t} / f_{c} \simeq 1.46 \sqrt{\epsilon}$ for small $\epsilon$. The explicit functional form of $f_{1}$ and $f_{2}$ can be obtained

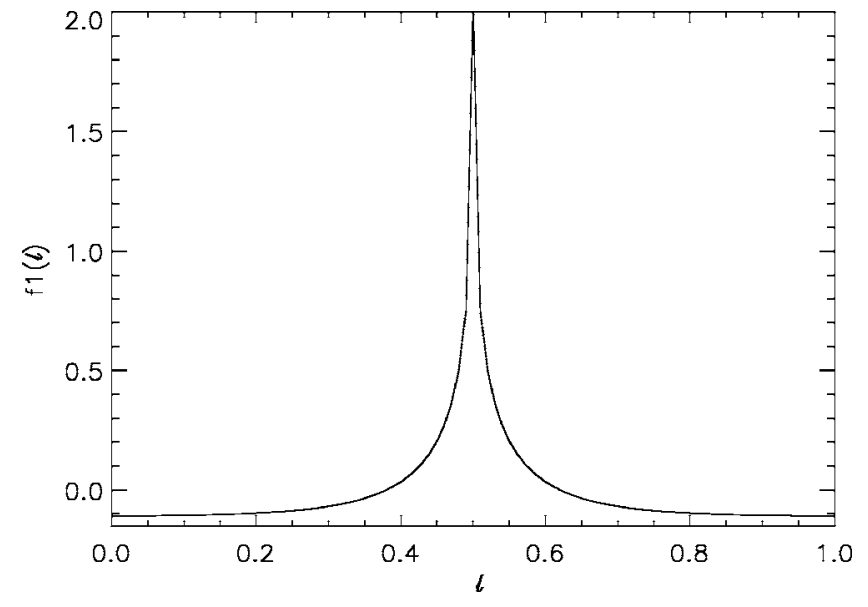

FIG. 2. The variation of $f_{1}$ as a function of $\theta$ corresponds to the largest term in $\mathbf{B} \cdot \nabla \cdot \boldsymbol{\Pi}_{\|} \sim \mathcal{O}\left(\epsilon^{-1}\right) f_{1}(\theta)$, for which $\left\langle f_{1}(\theta)\right\rangle=0$.

by introducing the expression for $f_{0}$ given by Eq. (36) in Eqs. (42) which yields

$$
\begin{aligned}
f_{1}= & -\frac{L}{1.46} \frac{\sqrt{2} \pi}{8}\left(1+\frac{1}{2} \frac{\sin (2 \pi \ell / L) E(\pi \ell / L, 1)}{\left[1-\sin ^{2}(\pi \ell / L)\right]^{3 / 2}}\right. \\
& -\int_{0}^{1} \frac{d s}{\left|1-s^{2} \sin ^{2}(\pi \ell / L)\right| E\left(s^{2}\right)} \\
& \left.\times\left\{1+\frac{3}{2} s^{2} \frac{\sin (2 \pi \ell / L) E\left(\pi \ell / L, s^{2}\right)}{\left[1-\sin ^{2}(\pi \ell / L)\right]^{3 / 2}}\right\}\right), \\
f_{2}= & \frac{1}{1.46} \frac{3 \pi \sqrt{2}}{8}\left\{\int_{0}^{1} \frac{d s}{E\left(s^{2}\right)} \frac{\sin (2 \pi \ell / L) E\left(\pi \ell / L, s^{2}\right)}{\left[1-s^{2} \sin ^{2}(\pi \ell / L)\right]^{3 / 2}}\right. \\
& -\frac{\sin (2 \pi \ell / L) E(\pi \ell / L, 1)}{\left.\sqrt{1-\sin ^{2}(\pi \ell / L)}\right\} .}
\end{aligned}
$$

Explicit evaluations of the functions in Eqs. (43) and (44) are shown in Figs. 2 and 3, respectively.

In the previous section it was shown that $\Pi_{\|}$in this model is a continuous function of $\ell$. However, its derivative

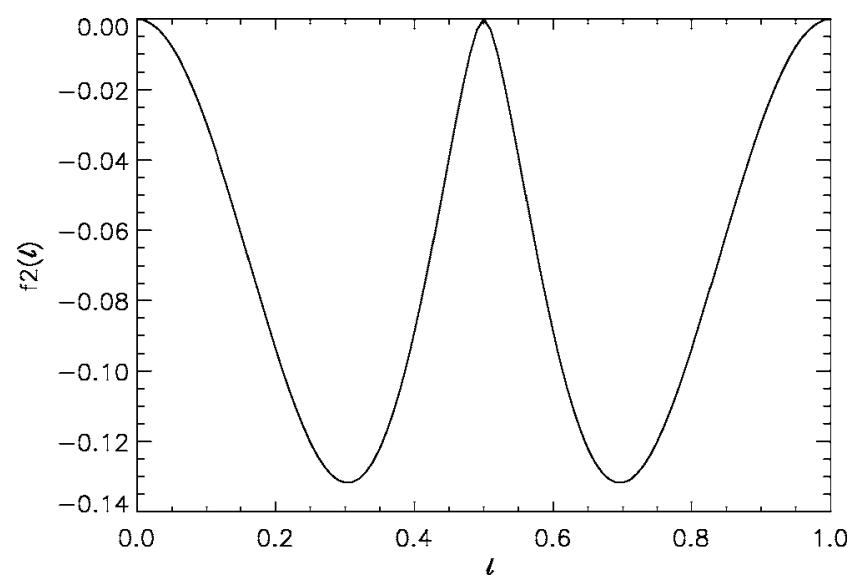

FIG. 3. The function $f_{2}$ gives the $\theta$ dependence of the lowest-order term in $\mathbf{B} \cdot \nabla \cdot \boldsymbol{\Pi}_{\|}$. This term has a nonzero flux-surface average and is thus the only term taken into account when $\left\langle\mathbf{B} \cdot \nabla \cdot \boldsymbol{\Pi}_{\|}\right\rangle$is calculated. 
in $\ell$ is singular at $\ell= \pm \pi / 2$ which is shown in Fig. 1 . In the pressure anisotropy $\Pi_{\|}$each term is singular but the order of the singularities (in the variable $\theta$ ) is the same and they cancel where the singularities in phase space at $\lambda=\lambda_{c}$ and real space $\theta= \pm \pi / 2$ coincide.

When one calculates the derivative of $\Pi_{\|}$in the realspace variable $\theta$ to obtain $f_{1}$, this balance is lost and the terms do not cancel anymore. However, as can be predicted from Fig. 1, we have

$$
\left\langle f_{1}(\ell)\right\rangle=0 .
$$

Because of this, as was discussed in Sec. II, the smallest term in Eq. (41) $\left(f_{2}\right)$ gives rise to the usual $\mathcal{O}(\sqrt{\boldsymbol{\epsilon}})$ result for $\left\langle\mathbf{B} \cdot \nabla \cdot \boldsymbol{\Pi}_{\|}\right\rangle$. For this term we have

$$
\left\langle f_{2}(\ell)\right\rangle=1,
$$

where the largest contribution to the average comes from the boundary term [second term in Eq. (41)] around the discontinuity in $\ell=L / 2$ :

$$
\lim _{\delta \rightarrow 0} \int_{L / 2-\delta}^{L / 2+\delta} \frac{d \ell}{L} \frac{\sin (2 \pi \ell / L) E(\pi \ell / L, 1) d \ell}{\left[1-\sin ^{2}(\pi \ell / L)\right]^{3 / 2}}=-1 .
$$

The divergence mentioned above arises from the fact that the boundary between trapped- and circulating-particle spaces is not well defined. That is, since particles with exactly $\lambda=\lambda_{c}$ are not either trapped or circulating, they cannot bounce back when they reach their turning point and neither can they move past this point. Thus, the time they spend there, which corresponds to $1 / \omega_{b}\left(\omega_{b} \rightarrow 0\right.$ for these particles), becomes infinite and the approximation $\nu_{*}$ $=\nu / \epsilon^{3 / 2} \omega_{b} \ll 1$ is not valid. Specifically, the derivatives of Eqs. (37) and (38) are

$$
\begin{aligned}
\frac{\partial}{\partial \theta} \frac{E\left(\pi \ell / L, s^{2}\right)}{\left[1-\sin ^{2}(\pi \ell / L)\right]^{3 / 2}} \sim & \frac{1}{\left|1-s^{2} \sin ^{2}(\pi \ell / L)\right|} \\
& +\frac{s^{2} \sin (2 \pi \ell / L) E\left(\pi \ell / L, s^{2}\right)}{\left[1-\sin ^{2}(\pi \ell / L)\right]^{5 / 2}} \\
& \left.\Rightarrow \ln x\right|_{x \rightarrow 0}
\end{aligned}
$$

and

$$
\left.\frac{\partial}{\partial \theta} \frac{E(\pi \ell / L, 1)}{\sqrt{1-\sin ^{2}(\pi \ell / L)}} \sim \frac{1}{\cos ^{2}(\pi \ell / L)} \Rightarrow \frac{1}{x^{2}}\right|_{x \rightarrow 0} .
$$

The term in Eq. (48) grows faster as $\ell \rightarrow \pm L / 2$ and the viscous force diverges as $1 / x^{2}$. A standard boundary layer procedure $^{3,7,17}$ at $\lambda \simeq \lambda_{c}$ will smooth the function at the singular points.

\section{HIGH-FREQUENCY LIMIT}

Before considering the complete frequency-dependent problem, the high-frequency limit is here calculated by neglecting the collisional term in the drift-kinetic equation (DKE). Taking a Laplace transform in Eq. (23) and assuming $i \omega / \nu \gg 1$, we obtain

$$
-i \omega \hat{F}+v_{\|} \mathbf{b} \cdot \nabla\left(\hat{F}+\frac{m}{T} v_{\|} B \hat{U} f_{M}\right)=\frac{v_{\|}}{p} f_{M} \mathbf{b} \cdot \widehat{\nabla \cdot \Pi_{\|}},
$$

where "hats" denote the Laplace-transformed quantities and $i \omega$ is the transform variable. Initial conditions are not taken into account here for simplicity. Thus, the solution is valid for short times but only after the initial conditions are damped.

As before, the source on the right-hand side of Eq. (49) does not contribute to the $P_{2}$ moment and is thus neglected. To lowest order $F$ is similar in structure to the one obtained for the equilibrium case. ${ }^{5}$ In this case the integration constant that arises when integrating the lowest-order equation is frequency dependent. Thus, we write $\hat{F}_{0}$ as

$$
\hat{F}_{0}=-\frac{m}{T} v_{\|} B \hat{U} f_{M}+\hat{g}(\psi, s, v, i \omega) .
$$

To determine $\hat{g}(\psi, s, v, i \omega)$, we consider the next order bounce-averaged equation $\left(-i \omega\left\langle F_{0} B / v_{\|}\right\rangle=0\right)$ which yields

$$
\hat{g}(\psi, s, v, i \omega)=\frac{m}{T} \hat{U} f_{M} \frac{\left\langle B^{2}\right\rangle}{\left\langle B / v_{\|}\right\rangle} .
$$

Proceeding as before, the next order equation is integrated along field lines. The integration constant in this case vanishes as is shown at the end of Appendix A. Since for trapped particles $\hat{g}=0, \hat{F}_{1}$ can be expressed as

$$
\hat{F}_{1}=-\frac{m}{T} \hat{U} f_{M}\left[B v_{\|}-\frac{\left\langle B^{2}\right\rangle}{\left\langle B / v_{\|}\right\rangle} \int_{0}^{\ell} \frac{d \ell}{v_{\|}} H\left(\lambda-\lambda_{c}\right)\right] .
$$

Changing the pitch-angle variable to $s$, given by Eq. (30), we can write the solution in terms of elliptic functions as follows:

$$
\hat{F}_{1}=-i \omega \frac{m}{T} \hat{U} f_{M}\left\{B \ell-\left\langle B^{2}\right\rangle \frac{L}{2} \frac{F\left(\pi \ell / L, s^{2}\right)}{K\left(s^{2}\right)} H\left(s^{2}-1\right)\right\} .
$$

Note that in this case, both the free-streaming term and the kinetic correction $\hat{g}$ are of the same order. This term $(B \ell)$ was neglected in the $\omega / \nu \ll 1$ case for which collisions have a more dramatic effect on the distribution function. When taking the corresponding pitch-angle integral of $\hat{F}_{1}$ for the stress or pressure anisotropy [Eq. (22)], the first term in Eq. (53) is calculated as follows:

$$
\begin{aligned}
\int_{0}^{1 / B} & \frac{B d \lambda}{v_{\|}}\left(\frac{3}{2} \lambda B-1\right) B \ell \\
= & \int_{0}^{1} \frac{B \ell s d s}{\left(s^{2}+2 \epsilon\right)^{3 / 2}} \frac{1}{\sqrt{1-s^{2} \sin ^{2}(\pi \ell / L)}} \\
& +B \ell \sqrt{1-\sin ^{2}(\pi \ell / L)},
\end{aligned}
$$

where the second term corresponds to the integration from the boundary at $s=1$ up to the turning point of the orbits at $s=\sin ^{-2}(\pi \ell / L)$. The pressure anisotropy in this highfrequency limit is thus 


$$
\begin{aligned}
\hat{\Pi}_{\|} \simeq & \frac{3 \sqrt{\epsilon}}{\sqrt{2}} i \omega m n \hat{U}\left\langle B^{2}\right\rangle\left\{\ell \sqrt{1-\sin ^{2}\left(\frac{\pi \ell}{L}\right)}\right. \\
& \left.-\int_{0}^{1} \frac{d s}{s^{2} \sqrt{1-s^{2} \sin ^{2}(\pi \ell / L)}}\left[\frac{L}{2} \frac{F\left(\pi \ell / L, s^{2}\right)}{K\left(s^{2}\right)}-\ell\right]\right\},
\end{aligned}
$$

from which the "drag-dominated" solution for the fluxsurface-averaged parallel viscous force $\left\langle\mathbf{B} \cdot \nabla \cdot \boldsymbol{\Pi}_{\|}\right\rangle \sim \mathcal{O}\left(\epsilon^{3 / 2}\right)$ (Ref. 18) is obtained. Once again, the function in Eq. (55) is continuous and remains finite for $\ell=0, n L / 2$.

\section{DYNAMIC PRESSURE ANISOTROPY}

In Secs. IV and V, we calculated both the low- and highfrequency limits for the parallel viscous force. It is desirable to have an expression for the pressure anisotropy in the general case. Keeping both the collisional and the time derivative terms in the DKE, we obtain the evolution of $F$ for any $\omega / \nu$ :

$$
-i \omega \hat{F}+v_{\|} \mathbf{b} \cdot \nabla\left(\hat{F}+\frac{m}{T} v_{\|} B \hat{U} f_{M}\right)-C(\hat{F})=\frac{v_{\|}}{p} f_{M} \mathbf{b} \cdot \widehat{\nabla \cdot \Pi}_{\|} .
$$

As in the previous sections, the expansion in $\nu_{*}$ for $F$ is introduced and the lowest-order equation is solved. The solution for the first term in the expansion can be expressed as $^{9,10}$

$$
\hat{F}_{0}=\left[-v_{\|} B+\frac{\bar{\nu}}{\hat{f}_{c}} \frac{v \varsigma}{2} \sum_{n=1}^{\infty} \frac{\eta_{n} \Lambda_{n}}{\left(\bar{\nu} \kappa_{n}-i \omega\right)}\right] \frac{m}{T} f_{M} \hat{U} .
$$

In Eq. (57) the $\lambda$-dependent functions are Cordey ${ }^{13}$ eigenfunctions which satisfy the following eigenvalue equation:

$$
\frac{d}{d \lambda}\left(\lambda\langle\sqrt{1-\lambda B}\rangle \frac{d \Lambda_{n}}{d \lambda}\right)=\kappa_{n} \Lambda_{n} \frac{d}{d \lambda}\langle\sqrt{1-\lambda B}\rangle,
$$

where $\kappa_{n}$ are the eigenvalues. The orthogonality condition is also in terms of flux-surface-averaged quantities and is given by

$$
\int_{0}^{\lambda_{c}} \Lambda_{n} \Lambda_{m} \frac{\partial\langle\sqrt{1-\lambda B}\rangle}{\partial \lambda} d \lambda=\delta_{n m} \int_{0}^{\lambda_{c}} \Lambda_{n}^{2} \frac{\partial\langle\sqrt{1-\lambda B}\rangle}{\partial \lambda} d \lambda .
$$

These functions have no exact analytical expression and are usually generated numerically. The quantities $\eta_{n}$ and $\hat{f}_{c}$ in Eq. (57) are defined in terms of $\Lambda_{n}$ and $\kappa_{n}$ :

$$
\begin{gathered}
\eta_{0}^{\lambda_{c}} \Lambda_{n} d \lambda \\
\int_{0}^{\lambda_{c}} \Lambda_{n}^{2}(\partial / \partial \lambda)\langle\sqrt{1-\lambda B}\rangle d \lambda \\
\hat{f}_{c} \equiv \hat{f}_{c}(\omega, v)=-\frac{3}{4}\left\langle B^{2}\right\rangle \sum_{n=1}^{\infty} \frac{\eta_{n}}{\kappa_{n}-i \omega / \bar{\nu}} \int_{0}^{\lambda_{c}} d \lambda \Lambda_{n} .
\end{gathered}
$$

Having the solution for the lowest-order distortion, the next order unaveraged DKE is integrated along field lines as was done before:

$$
\hat{F}_{1}=\int_{0}^{\ell} d \ell^{\prime} \frac{C\left(\hat{F}_{0}\right)}{v_{\|}}+i \omega \int_{0}^{\ell} d \ell^{\prime} \frac{1}{v_{\|}} \hat{F}_{0}+\frac{2}{3 B} \frac{f_{M}}{p} \Pi_{\|} .
$$

With the solution for the frequency-dependent $\hat{F}_{1}$, the pressure anisotropy can be calculated using Eq. (22). The contribution of the stress tensor vanishes upon calculating the corresponding moment as before. Using a small $\epsilon$ approximation, the dynamic pressure anisotropy can be written as

$$
\hat{\Pi}_{\|} \simeq \frac{1}{2 \epsilon} n m \hat{U} \sum_{1}^{\infty} I_{n}(\ell) \int d^{3} v \frac{\bar{\nu}}{v_{\mathrm{th}}^{2}} v^{2} \frac{f_{M}}{n} \frac{1}{\hat{f}_{c}} \frac{\eta_{n}}{\kappa_{n}-i \omega / \bar{\nu}},
$$

where the geometric coefficient $I_{n}(\ell)$ is given by

$$
\begin{aligned}
I_{n}(\ell)= & B\left(-\left.\frac{\zeta\left[\sqrt{1+\tau^{2}(\theta)}\right]}{\sqrt{1+\tau^{2}(\theta)}} \frac{\partial \Lambda_{n}}{\partial s}\right|_{s=1}+\int \frac{d s \Lambda_{n} s}{\left[1+s^{2} \tau^{2}(\theta)\right]^{3 / 2}}\right. \\
& \times\left\{s^{2} \zeta\left[\frac{\tau^{2}(\theta)}{\sqrt{1+s^{2} \tau^{2}(\theta)}}\right]\right. \\
& \left.\left.-\frac{2-s^{2} \tau^{2}(\theta)}{\left[1+s^{2} \tau^{2}(\theta)\right]^{3 / 2}} \zeta\left[\sqrt{1+s^{2} \tau^{2}(\theta)}\right]\right\}\right) .
\end{aligned}
$$

The expression for $I_{n}(\ell)$ for the bumpy cylinder magnetic field is

$$
\begin{aligned}
I_{n}(\ell)= & \frac{1}{4}\left\{\int_{0}^{1} \frac{s^{2} E(\pi \ell / L, s)}{\left[1-s^{2} \sin ^{2}(\pi \ell / L)\right]^{3 / 2}} \frac{\partial \Lambda_{n}}{\partial s} d s\right. \\
& \left.-\left.\frac{E(\pi \ell / L, 1)}{\sqrt{1-\sin ^{2}(\pi \ell / L)}} \frac{\partial \Lambda_{n}}{\partial s}\right|_{s=1}\right\},
\end{aligned}
$$

or, after integrating by parts,

$$
\begin{aligned}
I_{n}(\ell)= & -\frac{1}{4}\left\{\left.\frac{E(\pi \ell / L, 1)}{\sqrt{1-\sin ^{2}(\pi \ell / L)}} \frac{\partial \Lambda_{n}}{\partial s}\right|_{s=1}\right. \\
& -\int_{0}^{1} \frac{s \Lambda_{n}}{\left[1-s^{2} \sin ^{2}(\pi \ell / L)\right]^{3 / 2}}\left[\frac{3 E(\pi \ell / L, s)}{1-s^{2} \sin ^{2}(\pi \ell / L)}\right. \\
& \left.\left.-F\left(\frac{\pi \ell}{L}, s\right)\right] d s\right\} .
\end{aligned}
$$

The inverse Laplace transform of Eq. (63) cannot be obtained analytically. The denominator $\hat{f}_{c}$ is an infinite sum of integrals of the (numerically generated) Cordey eigenfunctions divided by $\kappa_{n}-i \omega / \bar{\nu}$ which also contains the corresponding eigenvalues. The poles of such an expression cannot be determined other than numerically.

Also, since both the eigenfunction equation and the orthogonality condition given in Eqs. (58) and (59) are in terms of flux-surface-averaged quantities, Eqs. (63) and (65) cannot be simplified any further. There is no trivial way to introduce the eigenvalues or to use the orthogonality condition to obtain a simplified form for $I_{n}(\ell)$. However, as pointed out before, the eigenfunctions can be generated numerically and 
introduced in Eq. (66) to calculate the inverse Laplace transform and obtain a numerical solution for the pressure anisotropy.

Equation (63) is valid for each flux surface despite the fact that it is written in terms of averaged quantities. The dependence in $\ell$ makes the expression vary within the flux surface. An analytical expression for the pressure anisotropy that depends only on $\ell$ and does not require the use of the flux-surface-averaged (Cordey) solutions could perhaps be obtained by developing a new set of unaveraged eigenfunctions as solutions to Eq. (56).

\section{SUMMARY}

In steady state the variation of the parallel viscous force was shown to be larger (by a factor of $1 / \epsilon$ ) than the averaged closure for a model magnetic field with small $|\mathbf{B}|$ modulations. To calculate the varying component, the first relevant correction to an equilibrium Maxwellian distribution function was calculated by integrating the first-order drift-kinetic equation along field lines in a steady-state situation and for small field modulations $(\epsilon \equiv \Delta B / 2 B \ll 1)$. With this result, the pressure anisotropy was obtained as a continuous functional of the distance along a field line [Eq. (35)] that, unlike in the collisional regime, is not simply sinusoidal but instead expressed in terms of incomplete elliptic functions.

The parallel viscous force was calculated in the steadystate case. As expected, its largest contribution $[\mathcal{O}(1 / \sqrt{\epsilon})]$ vanishes when a flux-surface average is calculated. This dominant term in the local closure has singularities at $B_{\max }$ for which a boundary layer procedure is suggested. The local closure obtained here, due to its larger magnitude, could modify the local plasma dynamics significantly compared to what is obtained with the averaged result. The short time limit for the pressure anisotropy was also calculated by neglecting collisions completely and the result, in which $\Pi_{\|}$ $\sim \mathcal{O}(\sqrt{\epsilon})$, coincides with the drag dominant solution for the parallel viscous force ${ }^{18}\left[\mathcal{O}\left(\epsilon^{3 / 2}\right)\right]$.

A generalization to a closure that varies within a flux surface and also depends on frequency was calculated and the result is expressed in terms of infinite sums of Cordey eigenfunctions. Equation (66) gives a formal solution to this problem but cannot be explored in general without the use of numerical tools. It could be worthwhile to pursue an analytical closure in terms of some other eigenfunction expansion that could be more easily handled to complete the analytic calculation. While the extension of this calculation to toroidal or other geometries is still to be done, we expect the essential scaling found in the bumpy cylinder model to be present in the more general case.

\section{ACKNOWLEDGMENT}

This research was supported by (U.S.) Department of Energy Grant No. DE-FG02-86ER53218.

\section{APPENDIX A: INTEGRATION CONSTANT}

When the second-order drift-kinetic-equation in Sec. III is integrated along a field line in general an integration constant $h(\psi, \lambda, v)$ is obtained:

$$
F_{1} \simeq \int_{0}^{\ell} \frac{C\left(F_{0}\right)}{v_{\|}} d \ell^{\prime}+\frac{2}{3 B} \frac{f_{M}}{p} \Pi_{\|}+h(\psi, \lambda, v) .
$$

To calculate $h(\psi, \lambda, v)$, consider the flux-surface average of Eq. (26),

$$
\begin{aligned}
\oint \frac{d \ell}{B} F_{1} \simeq & \oint \frac{d \ell}{B} \int_{0}^{\ell} \frac{d \ell^{\prime}}{v_{\|}} C_{R}\left(F_{0}\right)+\frac{2}{3 B} \frac{f_{M}}{p} \oint \frac{d \ell}{B} \Pi_{\|} \\
& +\oint \frac{d \ell}{B} h(\psi, \lambda, v) .
\end{aligned}
$$

Since the distribution function is periodic in $\ell$ and from Sec. II $\oint d \ell \prod_{\|} / B \sim \mathcal{O}(\sqrt{\epsilon})$, we have

$$
\oint \frac{d \ell}{B} h(\psi, \lambda, v)=-\oint \frac{d \ell}{B} \int_{0}^{\theta} \frac{d \ell^{\prime}}{v_{\|}} C_{R}\left(F_{0}\right)+\mathcal{O}(\sqrt{\epsilon}) .
$$

Using Eq. (32), with $\tau(\ell)=\sin ^{2}(\pi \ell / L)$, we obtain

$$
\begin{aligned}
\oint \frac{d \ell}{B} h(\psi, \lambda, v)= & \frac{U\left\langle B^{2}\right\rangle L}{B} f_{M} \frac{\nu_{\perp}}{v_{\mathrm{th}}^{2}} \oint \frac{d \ell}{B}\left\{1-\frac{E\left(\pi \ell / L, s^{2}\right)}{2 E\left(s^{2}\right)}\right. \\
& +\frac{1}{2 \epsilon} \frac{k^{2}}{2}\left[\frac{1}{2 E\left(s^{2}\right)} F\left(\frac{\pi \ell}{L}, s^{2}\right)\right. \\
& \left.\left.-\frac{E\left(\pi \ell / L, s^{2}\right)}{2 E^{2}\left(s^{2}\right)} K\left(s^{2}\right)\right]\right\},
\end{aligned}
$$

where all terms have been included. It can be easily seen that the second line vanishes when flux-surface averaged and thus

$$
h(\psi, \lambda, v)=\frac{\left\langle B^{2}\right\rangle L}{2 B} U f_{M} \frac{\nu_{\perp}}{v_{\mathrm{th}}^{2}} \sim \mathcal{O}(1) .
$$

Thus, since $\int_{0}^{\theta} d \theta C_{R}\left(F_{0}\right) / v_{\|} \sim \mathcal{O}\left(\epsilon^{-1}\right)$, the integration constant $h(\psi, \lambda, v)$ can be neglected.

For the high-frequency limit, also by bounce averaging Eq. (33), we find that the integration constant is given by

$$
\begin{aligned}
h(\psi, s, v) \oint \frac{d \ell}{v_{\|}} & =i \omega \frac{m}{T} U f_{M}\left[\oint \frac{B d \ell}{v_{\|}} \ell\right. \\
& \left.-\left\langle B^{2}\right\rangle \frac{L}{2} \frac{H\left(s^{2}-1\right)}{K\left(s^{2}\right)} \oint \frac{d \ell}{v_{\|}} \int_{0}^{\ell} \frac{d \ell^{\prime}}{v_{\|}}\right] .
\end{aligned}
$$

Since both integrals on the right are odd in $\ell$ they vanish and $h(\psi, s, v, i \omega)=0$.

\section{APPENDIX B: PRESSURE ANISOTROPY}

The pressure anisotropy can be calculated as 


$$
\begin{aligned}
\Pi_{\|} \sim & -\sqrt{2 \epsilon} m \sum_{\sigma} \pi \int_{0}^{\infty} d v v^{4} \\
& \times \int_{0}^{\sin ^{-2}(\theta)} \frac{s d s}{\left(s^{2}+2 \epsilon\right)^{3 / 2}} \frac{1}{\sqrt{1+s^{2} \tau(\theta)}} \int_{0}^{\theta} \frac{d \theta}{v_{\|}} C\left(F_{0}\right),
\end{aligned}
$$

from which the expression for the general case [Eq. (33)] is trivial to obtain assuming the first two terms in Eq. (32) can be neglected. The contribution of the free-streaming term to $\Pi_{\|}$is

$$
\int_{0}^{1 / B} \frac{B d \lambda}{v} \zeta\left(B^{2}\right) \simeq \frac{2}{\varsigma v} B_{0} \ell+\mathcal{O}(\sqrt{2 \epsilon}),
$$

which is $\mathcal{O}\left(\epsilon^{0}\right)$. The second term is, considering $\tau(\theta)$ $=\sin ^{2}(\pi \ell / L)$,

$$
\begin{aligned}
\int_{0}^{\lambda_{c}} \frac{d \lambda}{v_{\|}} \frac{\zeta\left(v_{\|}\right)}{\left\langle v_{\|}\right\rangle}= & -\frac{4 \sqrt{2 \epsilon}}{\varsigma v B_{0}^{2}} \int_{0}^{1} \frac{s d k}{\left(s^{2}+2 \epsilon\right)^{3 / 2}} \\
& \times \frac{1}{\sqrt{1-s^{2} \sin ^{2}(\pi \ell / L)}} \frac{E\left(\theta, s^{2}\right)}{2 E\left(s^{2}\right)} .
\end{aligned}
$$

In this case the integrand is singular at $s=0$. However, adding and subtracting the term

$$
\int_{0}^{1} \frac{s d s}{\left(s^{2}+2 \epsilon\right)^{3 / 2}} \simeq \frac{1}{\sqrt{2 \epsilon}}-1+\frac{2 \epsilon}{2},
$$

one can write Eq. (B3) as

$$
\begin{aligned}
\int_{0}^{\lambda_{c}} \frac{d \lambda}{v_{\|}} \frac{\zeta\left(v_{\|}\right)}{\left\langle v_{\|}\right\rangle} \\
\simeq \frac{2}{\varsigma^{\prime} B}\left(1-\sqrt{2 \epsilon}\left\{1-\int_{0}^{1} \frac{d s}{s^{2}}\left[\frac{L / \ell}{\sqrt{1-s^{2} \sin ^{2}(\pi \ell / L)}}\right.\right.\right. \\
\left.\left.\left.\quad \times \frac{E\left(\pi \ell / L, s^{2}\right)}{2 E\left(s^{2}\right)}-1\right]\right\}\right),
\end{aligned}
$$

which is also $\mathcal{O}(1)$. Thus, the combination of the first two terms is $\mathcal{O}(\sqrt{2 \epsilon})$ and can be neglected. Keeping only the last two terms in Eq. (32), we obtain

$$
\begin{aligned}
\Pi_{\|} \sim & \frac{1}{\sqrt{2 \epsilon}} \frac{L}{B_{0}} m \frac{\left\langle B^{2}\right\rangle}{4} U \sum_{\varsigma} \pi \int d v v^{4} f_{M} \frac{\nu_{\perp}}{v_{\mathrm{th}}^{2}} \\
& \times \int_{0}^{1} \frac{d s}{\sqrt{1+s^{2} \sin ^{2}(\pi \ell / L)}}\left[\frac{F\left(\pi \ell / L s^{2}\right)}{E\left(s^{2}\right)}\right. \\
& \left.-\frac{E\left(\pi \ell / L, s^{2}\right)}{E^{2}\left(s^{2}\right)} K\left(s^{2}\right)\right]
\end{aligned}
$$

from which Eq. (34) follows.

${ }^{1}$ F. L. Hinton and C. Oberman, Nucl. Fusion 9, 319 (1969).

${ }^{2}$ M. N. Rosenbluth, R. D. Hazeltine, and F. L. Hinton, Phys. Fluids 15, 116 (1972).

${ }^{3}$ F. L. Hinton and M. N. Rosenbluth, Phys. Fluids 16, 836 (1973).

${ }^{4}$ S. P. Hirshman and D. J. Sigmar, Nucl. Fusion 21, 1079 (1981).

${ }^{5}$ J. P. Wang and J. D. Callen, Phys. Fluids B 5, 3207 (1993).

${ }^{6}$ S. P. Hirshman, Nucl. Fusion 18, 917 (1978).

${ }^{7}$ F. L. Hinton and M. N. Rosenbluth, Plasma Phys. Controlled Fusion 41, 653 (1999)

${ }^{8}$ K. C. Shaing and S. P. Hirshman, Phys. Fluids B 1, 705 (1989).

${ }^{9}$ C. T. Hsu, K. C. Shaing, and R. Gormley, Phys. Plasmas 1, 132 (1994).

${ }^{10}$ A. L. García-Perciante, J. D. Callen, K. C. Shaing, and C. C. Hegna, Phys. Plasmas 12, 052516 (2005).

${ }^{11}$ R. C. Morris, M. G. Haines, and R. J. Hastie, Phys. Plasmas 3, 4513 (1996).

${ }^{12}$ E. D. Held, Phys. Plasmas 10, 4708 (2003).

${ }^{13}$ J. G. Cordey, Nucl. Fusion 16, 499 (1976).

${ }^{14}$ J. P. Wang and J. D. Callen, Phys. Fluids B 4, 1139 (1992).

${ }^{15} \mathrm{~S}$. Chapman and T. G. Cowling, The Mathematical Theory of NonUniform Gases (Cambridge University Press, Cambridge, 1939).

${ }^{16}$ A. L. García-Perciante, Ph.D. thesis, University of Wisconsin at Madison, 2005; CPTC Report No. UWPR 05-2, 2005.

${ }^{17}$ D. E. Baldwin and J. G. Cordey, Nucl. Fusion 12, 307 (1972).

${ }^{18}$ C. T. Hsu, K. C. Shaing, R. Gormley, and D. J. Sigmar, Phys. Fluids B 4, 12 (1992). 